\title{
Fractal Analysis for Symmetry Plane Detection in Neuroimages
}

\author{
Surani Anuradha Jayasuriya and Alan Wee-Chung Liew \\ School of Information and Communication Technology, \\ Griffith University, Gold Coast Campus, QLD, Australia \\ s.jayasuriya@griffithuni.edu.au, a.liew@griffith.edu. au
}

\begin{abstract}
Despite the considerable amount of research, brain symmetry plane detection is still an open problem. In this paper, we present a novel method for symmetry plane detection in magnetic resonance (MR) neuroimages based on the textural information and underlying brain's physiological structure. Fractal dimension and lacunarity analysis are used to locate the symmetry plane of the brain. The method was tested on MR data while analyzing the robustness against intensity non-uniformity, noise, and pathology. The proposed method does not need skull-stripping like pre-processing of MR images. The method was compared with another commonly used technique. The results were evaluated by an expert. The experimental results show the viability of our approach.
\end{abstract}

Keywords: fractal dimension, lacunarity, MRI, Mid-sagittal plane

\section{Introduction}

A normal brain is roughly symmetrical. Identifying the location of brain's symmetry plane is a key step in neuroimage analysis. It helps bring multiple images into a common coordinate system like Talairach-Tournoux [1] by reducing the degrees of freedom in multi-modal registration of brain images. Since an abnormality causes deviation from symmetry, brain symmetry plane also helps in diagnosis of various neurological disorders.

A deep furrow called the inter-hemispheric fissure separates the brain's left and right hemispheres (see Fig. 1). Although the real separation surface is not perfectly planar, a plane passes through this boundary is commonly called the mid-sagittal plane (MSP). The brain's symmetry plane is often considered as a first-order approximation to the MSP [2]. It is usually located manually by a neuroradiologist and taken as the reference plane for further analysis of the neuroimage. However, manual processing of a large number of images is a difficult and tedious task. Furthermore, since brain images varies from person to person and dependent on each scanner and modality, normalizing with respect to a standard brain image is difficult. An objective automated method for MSP detection in each image is advantageous. However, the natural complexity of brain and other artifacts in the image make MSP detection a challenging problem. 
A number of automatic methods have been proposed for detecting brain MSP [3]. However, most existing literature has focused on intensity based crosscorrelation without considering textural or structural information of the brain. In MRI, artifacts like intensity non-uniformity (INU) give rise to smooth intensity variation across the image. This intensity inhomogeneity or bias field can affect the accuracy of intensity based techniques [4,5]. Additionally, the structure and morphology of complex objects like brain are not well described by Euclidean geometry. The fractal geometry [6] is used to describe self-similar sets called fractals and natural objects that are difficult to describe by using the Euclidean geometry. It has been successfully applied in a variety of areas, including medical imaging [7]. However, the application of fractal analysis is often restricted to fractal dimension. Fractal dimension provides a quantitative index of the complexity or roughness of the object's structure [6]. Applications of the fractal dimension to medical images have been reported regarding quantitative texture analysis for novel understanding of brain structures and diagnosis of various diseases such as lesion and cancer ([8]-[11]). Kieselev et al. [12] has performed an analysis of the geometry of the brain cortex and their results indicate that the brain does possess some fractal property. However, fractal dimension alone is not sufficient to fully describe the space filling-characteristics of an object. Another fractal property called lacunarity describes the texture of a fractal object and can measure how the data fills the space [6]. Both fractal dimension and lacunarity are successfully used for classification of different structures and textures due to their invariance to scale, rotation or translation [11]. Since MR images have a degree of noise and randomness associated with the natural random texture of structure, they are said to be good candidates for characterization using fractal analysis [9]. In this work, we apply fractal dimension and lacunarity for identifying the symmetry plane or MSP in brain MRI.

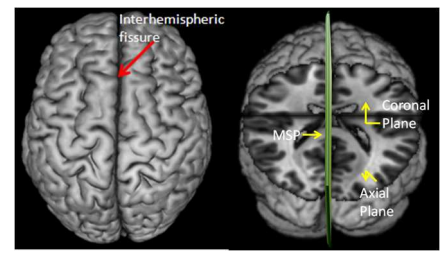

Fig. 1. Inter-hemispheric fissure/Mid-sagittal plane

\section{Related Work}

Existing work on MSP detection either depends on the similarity of the two brain hemispheres or the characteristics of an anatomical feature. In the former, MSP is defined as the plane that maximizes a symmetry criterion. In these methods ([3], [13]), the image is reflected across the initial plane and usually cross-correlation 
is used to measure the similarity between the original and the reflected images. Methods in this approach can easily be extended to different imaging modalities. However, because of the similarity criterion considered, these methods do not perform well when the brain image contains pathological asymmetry. Another disadvantage is the high computational cost. In the second approach ([14]-[15]), an anatomical feature is chosen (usually, the inter-hemispheric fissure) and then MSP is defined around it based on intensity properties. Since a similarity criterion is not used, these methods are generally insensitive to pathological asymmetry. However, methods in this approach are hard to extend to different imaging modalities and can be sensitive to noise and outliers.

Despite the variety of work that has been done in addressing the MSP detection problem, there is still no method commonly accepted as the best. In order to be useful in clinical setting, the method has to be fast, robust, accurate and automatic ([4], [14]). Existing techniques leave significant room for better applicability and accuracy. In our approach, we take into account the structural and textural differences of the region around the boundary between the two hemispheres and consider the MSP as the plane that best fits this boundary. In the brain, MSP contains larger amount of cerebrospinal fluid (CSF). In MR images, this appears as a clear textural difference. Since texture is a fundamental property that has been exploited to analyze tissues and pathologies, texture analysis using fractals could be used to differentiate structure along MSP from neighboring tissues.

\section{Methodology}

\subsection{Fractal Dimension}

Fractal dimension (FD) is the most frequently used fractal measure. It characterizes the irregularity and the complexity of an object. One criterion of a surface being fractal is its self-similarity [6]. A set $A$ is said to be self-similar when $A$ is the union of $N$ distinct copies of itself each of which has been scaled down by a ratio $r(r ; 1)$. Fractal dimension is defined as

$$
F D=-\frac{\ln (N)}{\ln (r)}
$$

The greater the $F D$ is the more complex and irregular the fractal object is. Therefore, FD can also be viewed as a measurement of the object's complexity or irregularity. There are a variety of algorithms to compute the FD of a structure. The basic procedure of the most commonly used box-counting (BC) method is to systematically lay a series of grids (boxes) of decreasing side length $r$ over an image and count the number $(N)$ of boxes with foreground pixels in them. $F D$ is then estimated using Eqn.1.

\subsection{Lacunarity}

When Mandelbrot introduced the fractal geometry, he defined and used the lacunarity as another complementary metric since the fractal dimension itself 
was not sufficient to fully capture the complexity of objects [6]. Lacunarity, from the Latin word lacuna meaning "gap" describes the texture of an object by measuring the degree of non-homogeneity within an object or image. If an object has large holes or gaps distributed unevenly, it has high lacunarity. On the other hand, if an object is homogeneous with respect to the spatial arrangement of gaps or almost translationally invariant, it has low lacunarity. Lacunarity has also been taken as a quantitative measure of texture in various fields. In [11], an approach based on lacunarity analysis on MR images is taken for studying trabecular bone structure to differentiate healthy and osteoporotic patients.

Several techniques have been proposed to estimate lacunarity. In the BC method, lacunarity $\lambda$ is calculated from the mean $(\mu)$ and the standard deviation $(\sigma)$ for the number of pixels within the box $r$ as

$$
\lambda=\frac{\sigma_{r}^{2}}{\mu_{r}^{2}}
$$

In brain MR images, MSP is visible as a distinguished pattern due to its higher amount of CSF. Going from one side towards the middle of the brain along the sagittal direction, the textural inhomogeneity increases towards the middle. This would increase the value of lacunarity (See Fig. 2). However, there is a possibility of having images with the same lacunarity value, but different fractal dimension due to structural change. Therefore, we need to use fractal dimension as well to fully consider the structural and textural information within the image. In any given axial slice, the complexity of the structure increases near the MSP (See Fig. 3). This would increase the fractal dimension. Therefore, our method is based on calculating the lacunarity of each sagittal slice and estimating the fractal dimension of a defined region-of-interest (ROI) on the axial images.

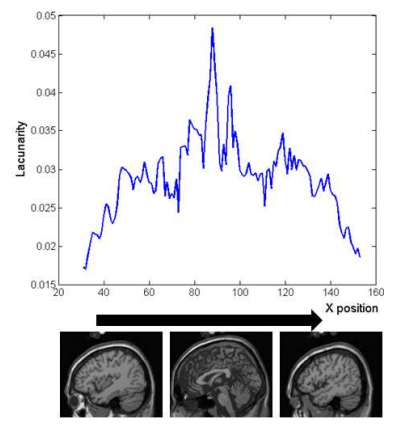

Fig. 2. Change of lacunarity value along the sagittal direction

\subsection{Procedure}

When pixel intensity is regarded as the height above plane, the intensity surface of a medical image can be regarded as a rough surface. We used a modified BC 
method called Differential Box Counting (DBC) [16] to estimate the FD as it is well-suited for computing fractal dimension of rough images. In this method, the image of size $M \times M$ pixels is partitioned into grids of size $s \times s$ where $M / 2 \geq s$ i 1 and $s$ is an integer. Then, the scaling down ratio is given by $r=s / M$. Consider the image $I(x, y)$ as a $2 \mathrm{D}$ plane and the third coordinate $(z)$ denoting gray level (See Fig. 3). On each image grid there is a stack of boxes of size $s \times s \times s^{\prime}$. If the total number of gray levels is $G$, then $s^{\prime}$ is chosen such that $s^{\prime} / G=r$. Assume that the maximum and minimum gray levels of the image in $(i, j)$ th grid fall in box number $k$ and $l$ respectively. Then, $n_{r}(i, j)=k-l+1$ is the contribution of $N_{r}$ in the $(i, j)$ th grid. The contributions from all the grids is obtained by

$$
N_{r}=\sum_{i, j} n_{r}(i, j)
$$

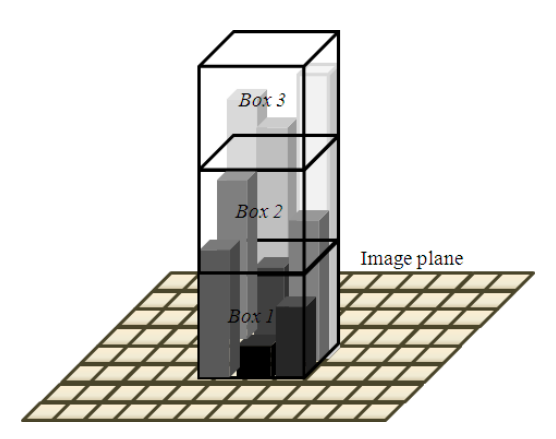

Fig. 3. Differential box counting with 3 boxes

The main steps of our algorithm are as follow:

1. Read the $3 \mathrm{D}$ brain volume data $I(x, y, z)$. Positive $X, Y, Z$ coordinates correspond to head's right, anterior and superior directions, respectively. Let the matrix size be $a \times b \times c$.

2. Generate a fractal image $I_{F D(x, y, z)}$ by applying the DBC method on the grayscale image. Since we are considering a $3 \mathrm{D}$ brain volume, we took pixel intensities as the fourth dimension. $I_{F D}$ remains the same for next two steps.

3. Working along the sagittal direction, compute the lacunarity $\lambda\left(x_{i}\right)$ for each sagittal slice position $x_{i}(1 ; i \leq a)$. Find the slice position $x_{i 0}$ that have the maximum lacunarity value $\left(x_{i 0}=\operatorname{argmax} \lambda\left(x_{i}\right)\right)$.

4. For Fine Processing: For each axial slice $j$ excluding the extreme top and bottom $20 \%$ slices; i.e., $(c / 5<j \leq 4 c / 5)$, define a ROI of a width of three pixel columns by centering on $x_{i 0}$. Then, compute the fractal dimension using a sliding window of a width of one pixel column inside the ROI. Find the location $p\left(j_{0}\right)$ with the maximum fractal dimension.

5. Estimate the MSP by taking the mode of $p\left(j_{0}\right)$ computed over the axial slices. 


\section{Results and Evaluation}

The MR image data were obtained from IBSR (http://www . cma.mgh . harvard. edu/) and BrainWeb (http://www.bic.mni.mcgill.ca/brainweb/). We considered $20 \mathrm{MR}$ scans of normal and pathological cases. T1-, T2- and PD-weighted images were included in the dataset. Simulated data from BrainWeb were used to analyze the effect of noise, INU, and image contrast. Fig. 4 shows some of the results obtained. Our results were visually evaluated by a medical expert and were judged to be consistently correct.
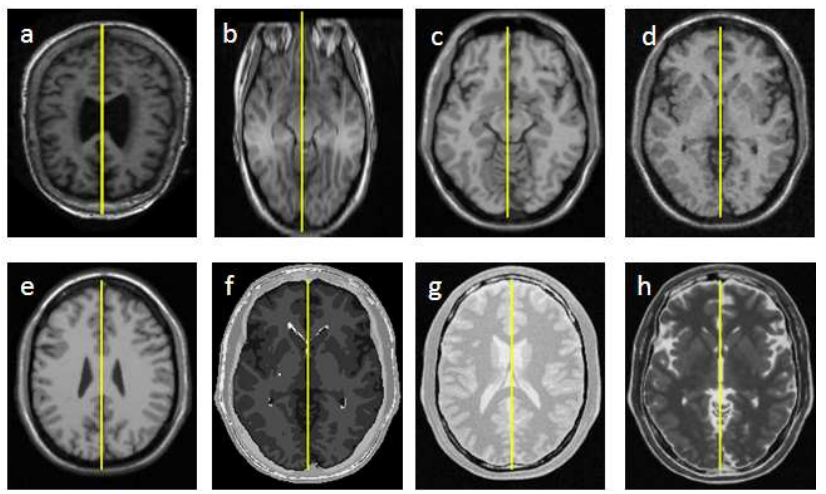

Fig. 4. Results for real and simulated images with various artifacts: (a) T1WI, (b)T1WI, (c)T1WI with added INU 20\%, (d)T1WI with added Noise 9\%, (e) T1WI, (f)T1WI-MS lesion, (g)PD, (d)T2WI.

Since cross-correlation (CC) is one of the most commonly used techniques in MSP detection, we compared the performance of our method with the CC technique. For each image we rotated our estimated line in 1 degree intervals from -5 to 5 and computed the intensity based $\mathrm{CC}$ of between the orginal image and its flipped version. The line that gave the maximum $\mathrm{CC}$ was compared with the line estimated by our method. As shown in Fig. 5, despite its slightly lower $\mathrm{CC}$ value our approach gave better results. For a quantitative comparison, we measured the angular deviation ( $\alpha$, in degrees) and the deviation of the Euclidean distance ( $d$, in millimeters) of the end points between each method's estimated MSPs and the ground truth lines. Summary of the results are shown in Table 1.

Table 1. Comparison with the cross-correlation technique

\begin{tabular}{|c||cccc|}
\hline Methods & $\alpha_{\text {mean }}$ & $\alpha_{\text {stdev }}$ & $d_{\text {mean }}$ & $d_{\text {stdev }}$ \\
\hline Our method & 0.4 & 0.39 & 0.32 & 0.47 \\
CC Technique & 1.3 & 0.48 & 0.77 & 0.65 \\
\hline
\end{tabular}



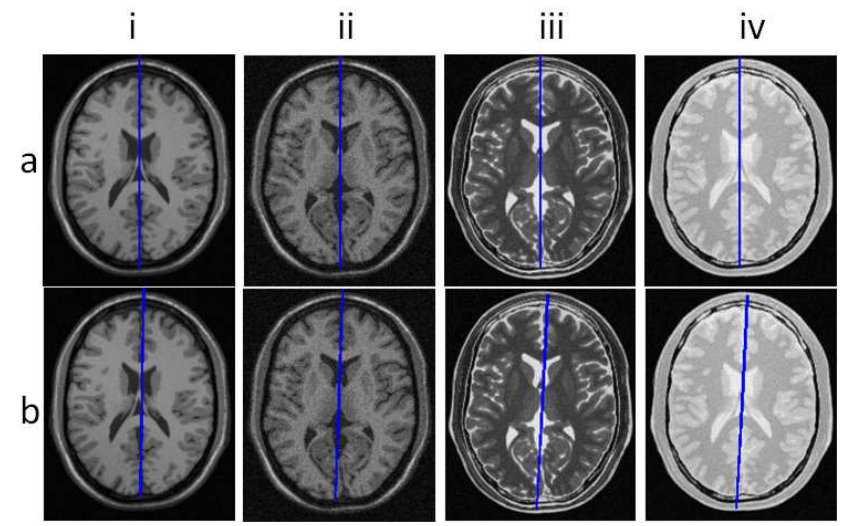

Fig. 5. Comparison of the proposed method with CC method on MRI with varying artifacts and contrast: (a). Our method. (b). CC method. (i). T1-INU40\%, (ii). T1Noise9\%, (iii). T2, (iv), PD.

As shown in Fig. 5, our method was robust to noise, INU and different modalities, while CC method was sensitive to these factors. We assume this is due to the fact that our method does not rely on a maximization of pixel-wise grayscale similarity of the two hemispheres. Instead, we compare the structural complexity and the homogeneity by using fractal dimension and lacunarity. Moreover, when the $\mathrm{CC}$ as a similarity measure is performed slice-by-slice, it gives less accurate results if the given neuroimage is strongly tilted, as then the left-right similarity breaks.

\section{Conclusion}

We have presented a novel approach based on fractal analysis for detecting the symmetry plane in MR neuroimages. In contrast to many existing methods that measure the similarity of the two brain hemispheres using intensity-based crosscorrelation, our method assumes that the MSP has the most complex and inhomogeneous structure amongst all sagittal slices and this structural complexity shows up as a textural difference on MR images. We have used a combination of fractal dimension and lacunarity as a relative measure to characterize the structural complexity associated with the mid-sagittal plane. While similarity based methods could be severely affected by pathologies and hemispheric dissimilarity due to tilted head scans, our method is less sensitive to such asymmetry.

The proposed method has been tested on various MR images and is robust to different imaging modalities, noise and INU artifacts. Experimental results show the effectiveness of our approach. In comparison with the most frequently used cross-correlation method, the performance of our fractal analysis based method is very encouraging. 
Acknowledgments. This work was supported by the Australian Research Council (ARC) Discovery Grant DP1097059 and the Australian Postgraduate Award (APA).

\section{References}

1. Talairach, J., Tounoux, P.: Co-Planar Stereotaxic Atlas of the Human Brain: 3D Proportional System: An Approach to Cerebral Imaging. Thieme Medical, New York (1988)

2. Kruggel, F., von Cramon, D.: Alignment of Magnetic Resonance Brain Datasets with the Stereotactical Coordinate System. Medical Image Analysis 3(2), 175-185 (1999)

3. Prima, S., Ourselin, S., Ayache, N.: Computation of the Mid-Sagittal Plane in 3D Brain Images. IEEE Transactions on Medical Imaging 21, 122-138 (2001)

4. Liew, A.W.C., Yan, H.: Current Methods in the Automatic Tissue Segmentation of 3D Magnetic Resonance Brain Images. Current Medical Imaging Reviews 2(1), 91-103 (2006)

5. Liew, A.W.C., Yan, H.: An Adaptive Spatial Fuzzy Clustering Algorithm for MR Image Segmentation. IEEE Transactions on Medical Imaging 22(9), 1063-1075 (2003)

6. Mandelbrot, B.: The Fractal Geometry of Nature. W.H. Freeman and Company, (1982)

7. Lopes, R., Betrouni, N.: Fractal and Multifractal Analysis: A Review. Medical Image Analysis 13, 634-649 (2009)

8. Blackledge, J., Dubovitskiy, D.: Object Detection and Classification with Applications to Skin Cancer Screening. ISAST Transactions on Intelligent Systems 1(2), $34-45$ (2008)

9. Iftekharuddin, K.: Techniques in fractal analysis and their applications in brain MRI. Medical imaging systems: technology and applications, Analysis and Computational Methods 1, 63-86 (2005)

10. Takahashi, T., Kosaka, H., Murata, T., Omori, M., Nartia, K., Mitsuya, H., Takahashi, K., Kimura, H., Wada, Y. : Application of a Multifractal Analysis to Study Brain White Matter Abnormalities of Schizophrenia on T2-weighted Magnetic Resonance Imaging. Psychiatry Research Neuroimaging 171, 177-188 (2009)

11. Zaia, A., Eleonori, R., Maponi, P., Rossi, R., Murri, R. : MR Imaging and Osteoporosis: Fractal Lacunarity Analysis of Trabecular Bone. IEEE Transactions on Information Technology in Biomedicine 10(3), 484-489 (2006)

12. Kiselev, V., Hahn, K., Auer, D.: Is the Brain Cortex a Fractal?. Neuroimage 20, 1765-1774 (2003)

13. Liu, Y., Collins, R., Rothfus, W.: Robust Midsagittal Plane Extraction from Normal and Pathological 3-D Neuroradiology Images. IEEE Transactions on Medical Imaging 20(3), 175-192 (2001)

14. Hu, Q., Nowinski, W.: A rapid algorithm for robust and automatic extraction of the midsagittal plane of the human cerebrum from neuroimages based on local symmetry and outlier removal. Neuroimage 20, 2153-2165 (2003)

15. Volkau, I., Bhanu, P., Ananthasubramaniam, A., Aziz, A., Nowinski, W.: Extraction of the Midsagittal Plane from Morphological Neuroimages using the KullbackLeibler's Measure. Medical Image Analysis 10, 863-874 (2006)

16. Sarkar, N., Chaudhuri, B.: An Efficient Differential Box-Counting Approach to Compute Fractal Dimension of Image. IEEE Transactions on Systems, Man and Cybernetics 24(1), 115-120 (1994) 